\title{
A blind and independent benchmark study for detecting differentially methylated regions in plants
}

\author{
Clemens Kreutz ${ }^{1,2, *}$, Nilay S. Can ${ }^{3}$, Ralf Schulze Bruening ${ }^{3}$, Rabea Meyberg ${ }^{3}$, \\ Zsuzsanna Mérai ${ }^{4}$, Noe Fernandez-Pozo ${ }^{3}$ and Stefan A. Rensing ${ }^{3,5}$
}

${ }^{1}$ Faculty of Medicine and Medical Center, Institute of Medical Biometry and Statistics, University of Freiburg, 79104 Freiburg, Germany, ${ }^{2}$ Centre for Integrative Biological Signalling Studies (CIBSS), University of Freiburg, 79104 Freiburg, Germany, ${ }^{3}$ Plant Cell Biology, Faculty of Biology, University of Marburg, 35043 Marburg, Germany, ${ }^{4}$ Gregor Mendel Institute of Molecular Plant Biology, Austrian Academy of Sciences, Vienna BioCenter (VBC), 1030 Vienna, Austria and ${ }^{5}$ Centre for Biological Signaling Studies (BIOSS), University of Freiburg, 79104 Freiburg, Germany

*To whom correspondence should be addressed.

Associate Editor: Alfonso Valencia

Received on August 20, 2019; revised on January 31, 2020; editorial decision on March 12, 2020; accepted on March 13, 2020

\begin{abstract}
Motivation: Bisulfite sequencing (BS-seq) is a state-of-the-art technique for investigating methylation of the DNA to gain insights into the epigenetic regulation. Several algorithms have been published for identification of differentially methylated regions (DMRs). However, the performances of the individual methods remain unclear and it is difficult to optimally select an algorithm in application settings.

Results: We analyzed BS-seq data from four plants covering three taxonomic groups. We first characterized the data using multiple summary statistics describing methylation levels, coverage, noise, as well as frequencies, magnitudes and lengths of methylated regions. Then, simulated datasets with most similar characteristics to real experimental data were created. Seven different algorithms (metilene, methylKit, MOABS, DMRcate, Defiant, BSmooth, MethyISig) for DMR identification were applied and their performances were assessed. A blind and independent study design was chosen to reduce bias and to derive practical method selection guidelines. Overall, metilene had superior performance in most settings. Data attributes, such as coverage and spread of the DMR lengths, were found to be useful for selecting the best method for DMR detection. A decision tree to select the optimal approach based on these data attributes is provided. The presented procedure might serve as a general strategy for deriving algorithm selection rules tailored to demands in specific application settings.

Availability and implementation: Scripts that were used for the analyses and that can be used for prediction of the optimal algorithm are provided at https://github.com/kreutz-lab/DMR-DecisionTree. Simulated and experimental data are available at https://doi.org/10.6084/m9.figshare.11619045.
\end{abstract}

Contact: ckreutz@imbi.uni-freiburg.de

Supplementary information: Supplementary data are available at Bioinformatics online.

\section{Introduction}

A large number of methods and algorithms have been established during recent years for analyzing high-throughput data and often several approaches exist for the same task. Because the performance of such competing algorithms is usually context-specific (Shippy et al., 2006; Su et al., 2014; Webb-Robertson et al., $2015)$, i.e. depends on characteristics of the investigated biological system as well as on the amount and quality of the data, clear and general rules for selecting the most suitable algorithm in practice are missing. Therefore, benchmark studies for deriving such guidelines are essential. In this study, we compared multiple tools to detect differentially methylated regions (DMRs) in plant bisulfite sequencing data (BS-seq, also known as Bisulfite-Seq or Methylseq).

DNA methylation can have a remarkable effect on gene expression and cellular activity and analyzing it via deep sequencing is an important approach to study its impact on transcription. BS-seq is the most common method to study DNA methylation to nucleotide-level. BS-seq can be combined with long-read sequencing for real-time bisulfite sequencing (Yang and Scott, 2017). Other methods, such as ChIP-seq exists to study histone modifications (Chen et al., 2018), and novel methods, such as TET-assisted pyridine borane sequencing, could be the future for investigation of 
DNA methylation at nucleotide level since it is a cheaper and more reliable alternative to BS-seq (Liu et al., 2019). In BS-seq, to identify the DNA methylation status, DNA samples are treated with sodium bisulfite before sequencing. This way, methylated cytosines (including 5-Methylcytosine and 5-Hydroxymethylcytosine) remain unchanged, whereas unmethylated cytosines are converted to uracils.

For the detection of DMRs, many different approaches are available (Robinson et al., 2014), and in our application setting, it was unclear how to optimally choose from these competing methods. Existing studies provide a heterogeneous and inconsistent picture, with limited information for the usage on plant BS-seq data where, in contrast to e.g. mammals, DNA methylation can be observed not only in $\mathrm{CG}$ context but also in $\mathrm{CHG}$ and $\mathrm{CHH}$ contexts $\mathrm{H}=\mathrm{A}, \mathrm{C}$ or T) (Sahu et al., 2013). In addition, DMR algorithms tested in plants, are often limited to Arabidopsis thaliana, but DNA methyltransferases, genome size, methylation patterns and transposable elements differ between plant species (Bewick and Schmitz, 2017; Lang et al., 2008). Thus, studying multiple species is important.

Based on recent DMR benchmark studies it is not evident which algorithms are most suitable for DMR detection in plants. For example, Jühling et al. (2016) introduced metilene and compared it with three other DMR algorithms: MOABS (Sun et al., 2014), BSmooth (Hansen et al., 2012) and BiSeq (Hebestreit et al., 2013) using experimental datasets from human and simulated datasets based on human Chromosome 10. Metilene showed superior specificity, sensitivity and accuracy and particularly outperformed the others when the methylation background was variant and the methylation differences were subtle. A similar benchmark is done with another tool, Defiant (Condon et al., 2018) comparing it with six other DMR tools: BSmooth, methylKit (Akalin et al., 2012), MethylSig (Park et al., 2014), metilene, MOABS and RADMeth (Dolzhenko and Smith, 2014), using the simulated data from Jühling et al. (2016) and experimental BS-seq data from rat. Defiant showed comparable performance with metilene for simulated data and identified more DMRs in the experimental dataset; however, several low coverage DMRs were only detected by metilene. In Gong and Purdom (2018), MethCP was presented in comparison with BSmooth, HMM-Fisher, DSS, methylKit and metilene in an analysis of simulated datasets. Here, MethCP and metilene showed the highest rate of true positives among all the tools tested. In Catoni et al. (2018), experimental data from human cell lines, rice and A.thaliana were analyzed to assess the performance of DMRcaller in comparison with methylKit and MethylSig, resulting in better performance of DMRcaller. In all of the studies aforementioned, superior performance of the newly presented approach was concluded, presenting only partially consistent outcomes as compared with previous studies.

The heterogeneity of the outcomes might originate from the fact that different types of data with distinct characteristics were used for assessment. Most DMR analyses are based on human or mammals and on the $C G$ methylation context, consequently ignoring plant methylation contexts $\mathrm{CHH}$ and $\mathrm{CHG}$. Only two of the previous benchmark studies (Catoni et al., 2018; Gong and Purdom, 2018) partly focused on DMRs in plants. However, the designs of both studies were not based on typical data and they do not assess the tradeoff between true positives and false positives. In Catoni et al. (2018), wild-type was compared with knockouts of methyltransferases. These knockouts are known to prevent methylation and, therefore, only sensitivity could be evaluated by treating all DMR predictions exclusively as true positives. In Gong and Purdom (2018), random permutations are applied to generate uninformative data and then only specificity was assessed by treating all predictions as false positives.

Different plant groups differ in their DNA methyltransferase complements and context-specific DNA methylation (Bewick et al., 2017). Here, we include data of the prime plant model, A.thaliana, and of a relative from the same order (Brassicales), Aethionema arabicum, representing low coverage data (Table 1). We also include data of Picea abies, in which methylation frequencies are different (Heer et al., 2018) and of a moss, Physcomitrella patens, which again shows differences, namely different properties of gene body methylation (Lang et al., 2018).

In this study, we, therefore, intended to analyze realistically simulated data including multiple plant species from several plant groups, i.e. a bryophyte (patens), a gymnosperm (P.abies) and two angiosperms (Ae.arabicum and A.thaliana). All of these datasets are again different from those of mammals, in both terms, methylation context (including $\mathrm{CHG}$ and $\mathrm{CHH}$ contexts) and distribution of methyl groups. As described in the following, we took experimental data from these plants as templates and calibrated a simulation framework for generating data with attributes most similar to our experimental datasets. We evaluated the performance of BSmooth,

Table 1. Overview about the experimental BS-seq datasets

Abbreviation Description

DNA Analyzed Analyzed Analyzed Coverage context region range (bp) data points

\begin{tabular}{|c|c|c|c|c|c|c|c|}
\hline \multirow{21}{*}{ Data context } & Aetar-C-CG & Ae.arabicum, Cyprus ecotype, seeded at $20^{\circ} \mathrm{C}$ & CG & Scaffold 65 & 2720010 & 29356 & 3.11 \\
\hline & Aetar-C-CHG & Ae.arabicum, Cyprus ecotype, seeded at $20^{\circ} \mathrm{C}$ & $\mathrm{CHG}$ & Scaffold 65 & 2725648 & 48748 & 2.29 \\
\hline & Aetar-C-CHH & Ae.arabicum, Cyprus ecotype, seeded at $20^{\circ} \mathrm{C}$ & $\mathrm{CHH}$ & Scaffold 65 & 2728141 & 214994 & 2.11 \\
\hline & Aetar-c-CG & Ae.arabicum, Cyprus ecotype, seeded at $25^{\circ} \mathrm{C}$ & CG & Scaffold 65 & 2720259 & 27989 & 3.26 \\
\hline & Aetar-T-CHG & Ae.arabicum, Turkey ecotype, seeded at $20^{\circ} \mathrm{C}$ & $\mathrm{CHG}$ & Scaffold 65 & 2730515 & 59415 & 2.60 \\
\hline & Aetar-T-CHH & Ae.arabicum, Turkey ecotype, seeded at $20^{\circ} \mathrm{C}$ & $\mathrm{CHH}$ & Scaffold 65 & 2731240 & 266096 & 2.36 \\
\hline & Arath-CG & A.thaliana, root tissue & CG & Chr 2 & 19696777 & 903767 & 34.72 \\
\hline & Arath-CHG & A.thaliana, root tissue & CHG & Chr 2 & 19696771 & 977493 & 33.96 \\
\hline & Arath-CHH & A.thaliana, root tissue & $\mathrm{CHH}$ & Chr 2 & 3952731 & 1000000 & 31.36 \\
\hline & Picab-M-GG & P.abies, $500 \mathrm{~m}$ above sea level & CG & 50 scaffolds & 2822478 & 33101 & 41.38 \\
\hline & Picab-M-CHG & P.abies, $500 \mathrm{~m}$ above sea level & $\mathrm{CHG}$ & 26 scaffolds & 1554819 & 61918 & 21.00 \\
\hline & Picab-M-CHH & P.abies, $500 \mathrm{~m}$ above sea level & $\mathrm{CHH}$ & 3 scaffolds & 243324 & 61918 & 14.53 \\
\hline & Picab-P-GG & P.abies, $1200 \mathrm{~m}$ above sea level & CG & 50 scaffolds & 2822266 & 32967 & 30.86 \\
\hline & Picab-P-CHG & P.abies, $1200 \mathrm{~m}$ above sea level & $\mathrm{CHG}$ & 26 scaffolds & 1586240 & 61918 & 17.01 \\
\hline & Picab-P-CHH & P.abies, $1200 \mathrm{~m}$ above sea level & $\mathrm{CHH}$ & 3 scaffolds & 246862 & 61918 & 11.90 \\
\hline & \multicolumn{2}{|c|}{ Phypa-G-CG patens, Gransden ecotype } & CG & Chr 27 & 5294665 & 182243 & 11.32 \\
\hline & \multicolumn{2}{|c|}{ Phypa-G-CHG patens, Gransden ecotype } & $\mathrm{CHG}$ & Chr 27 & 5295410 & 200348 & 10.74 \\
\hline & \multicolumn{2}{|c|}{ Phypa-G-CHH patens, Gransden ecotype } & $\mathrm{CHH}$ & Chr 27 & 5298384 & 1347668 & 8.02 \\
\hline & Phypa-R-CG & patens, Reute ecotype & CG & Chr 27 & 5294552 & 178799 & 12.12 \\
\hline & Phypa-R-CHG & patens, Reute ecotype & $\mathrm{CHG}$ & Chr 27 & 5295374 & 197297 & 12.25 \\
\hline & Phypa-R-CHH & patens, Reute ecotype & $\mathrm{CHH}$ & Chr 27 & 5298334 & 1330264 & 8.67 \\
\hline
\end{tabular}

Note: Representative genomic regions were analyzed to characterize attributes of the data and for optimizing the simulation parameters. The provided coverage is the average number of reads of the analyzed genomic positions. For each data context, 1000000 data points were simulated. 
Defiant, DMRcate (Peters et al., 2015), metilene, methylKit, MethylSig and MOABS. Other tools, such as BiSeq (Hebestreit et al., 2013), BEAT (Akman et al., 2014), DSS (Feng et al., 2014), RnBeads (Assenov et al., 2014), M3D (Mayo et al., 2015), were not included because it has been claimed that they cannot handle methylation in non-CG context (Catoni et al., 2018).

Recently, neutral benchmark studies that do not focus on introducing a new method and guarantee that the authors have no preferences have been demanded (Boulesteix et al., 2017). Moreover, knowledge about the underlying truth provides information for the definition of configuration parameters that is not available in real experimental setting and thus might lead to overoptimistic assessment. To avoid such biases, we chose a blinded study designs, i.e. data simulations and assessments were strictly separated from the DMR analyses. Based on this blinded study design, we derived a decision tree that can serve as algorithm selection rule in new applications.

\section{Materials and methods}

BS-seq data of four plant species from different taxonomic groups were used to create simulated realistic datasets to evaluate the performance of seven DMR prediction tools. Conditions and characteristics of the methylation data from these experimental samples are summarized in Table 1.

\subsection{Methylation data}

For Ae.arabicum (Brassicaceae), representing one of the angiosperm species from this study, dried seed samples of two different ecotypes (Turkey and Cyprus) were used to create simulated datasets (for sample preparation see Supplementary information). Arabidopsis thaliana, another Brassicaceae, was selected to be analyzed in this study as the most well-studied plant model. To create the simulated datasets for A.thaliana, methylation call files from bisulfite-treated root samples from Seymour et al. (2014) were used. Data from P.abies were selected to represent gymnosperms. Methylation calls from Heer et al. (2018) of trees situated at about 1200 meters above sea level (MASL) and clones from those trees, planted 24 years ago at 520 MASL were used to create the simulated data. patens was selected as a model plant to represent bryophytes. Simulated datasets were generated using methylation calls derived from adult gametophores of two different ecotypes, Gransden (Lang et al., 2018) and Reute (Meyberg et al., 2019). Twenty-one BS-seq datasets of four plant species in three methylation contexts (CG, CHG and $\mathrm{CHH}$ ) were selected for benchmarking. These datasets differ in their sequencing depth, from very low $(<5 \times)$ in Ae.arabicum, low $(10 \times)$ in patens, medium $(10-20 \times)$ in P.abies and high $(30 \times)$ in A.thaliana (Table 1). The raw data from A.thaliana, P.abies and patens are available in the Sequence Read Archive with accession numbers: PRJEB6701, PRJEB26494, SRR4454535 and SRR9901085. The scaffold 65 data used for Ae.arabicum is available at https://doi.org/10.6084/m9.figshare.11619045.

\subsection{DMR algorithm configuration}

BSmooth v1.18, Defiant v1.1.3, DMRcate v1.18, methylKit v1.8.1, MethylSig v0.5.2, metilene v0.2.7 and MOABS v1.3.2 were used to predict DMR for all simulated datasets. Tools were used with default parameters and when possible, $P$-value thresholds were set to 0.05 , the minimum methylation difference was set to $10 \%$, the window size was set to $200 \mathrm{bp}$ for tools using windows to calculate DMRs, and the minimum coverage was set to 3 for all tools and datasets, with the following exceptions. Metilene has no option to filter by minimum coverage, so minimum coverage was 0 . Defiant was not able to produce results for Ae.arabicum datasets, with very low coverage, so the minimum coverage was set to 0 in these cases and 3 was used for all other cases.

A modified version of metilene v0.2.7 was provided by the developers to be able to process $\mathrm{CHH}$ and $\mathrm{CHG}$ contexts. This version included a parameter $(\mathrm{G})$ for the chunk size to prevent software crashes due to the high frequency of $\mathrm{CHH}$ and $\mathrm{CHG}$ patterns. The chunk size was set to 200 for all analyses using metilene for the contexts $\mathrm{CHH}$ and $\mathrm{CHG}$. The chunk size parameter was included in metilene v0.2.8.

\subsection{Simulating data}

Simulated data were generated and utilized to assess the performances of seven DMR identification methods. For data simulation, we utilized the WGBSSuite, a software package that has been developed for simulated whole genome bisulfite sequencing data (Rackham et al., 2015).

The WGBSSuite simulates the data by the following procedure. In a first step, a discrete-state Markov model is used to subdivide genomic regions into CG islands and deserts. The $2 \times 2$ transition matrix $T$ has two free parameters $T_{12}$ for the transition probabilities from desert to islands and $T_{21}$ from $C G$ islands to deserts. The other two entries of the transition matrix $T_{11}$ and $T_{22}$ are given by normalization, i.e. $T_{11}=1-T_{12}$ and $T_{22}=1-T_{21}$. Each simulation is initialized in the desert state.

In the second step, the methylation sites are drawn from exponential distributions. The distance of subsequent sites simulated according to $\Delta$ pos $=\max \{1, \operatorname{round}(\Delta x)\}$ with $\operatorname{Prob}(\Delta x)=(1 / R) \mathrm{e}^{-\Delta x / R}$ for $\Delta x>0$. In the classical setting, these sites correspond to consecutive CG locations. For our setup in plants, the sites may also correspond to $\mathrm{CHG}$ or $\mathrm{CHH}$, depending on the analyzed context. Different densities for $C G$ islands and deserts are defined by two rates $R=R_{\text {island }}$ or $R=R_{\text {desert }}$. The larger these rates, the closer are the simulated positions of the methylation sites.

As a third step, the methylation status is simulated. Here, a nonhomogeneous HMM is used and the transitions between states are modulated by the distances of the methylation sites. The simulation suite describes methylation via four states, an unmethylated state $S_{1}$, a transition state from unmethylated to methylated $S_{2}$, a methylated state $S_{3}$ and a transition state $S_{4}$ from methylated to unmethylated. There is a $4 \times 4$ transition matrix $\Pi$ that does only allow transitions in plausible order $S_{1} \rightarrow S_{2} \rightarrow S_{3} \rightarrow S_{4}$ and, therefore, has four offdiagonal elements $\Pi_{12}, \Pi_{23}, \Pi_{34}, \Pi_{41}$. In our analyses, the transition rates from the intermediate states $S_{2}$ and $S_{4}$ were fixed to the default value $\Pi_{23}=\Pi_{41}=0.02$, the two additional off-diagonal elements $\Pi_{12}$ and $\Pi_{34}$ controlling transitions, i.e. the lengths of the methylated and unmethylated regions, were estimated from the experimental data as discussed in the following section. This four-state HMM describes the methylation changes between consecutive genomic sites. The dependency on the distances of consecutive sites is included by a decay of the probabilities for remaining in a state, i.e. the decrease in diagonal elements is multiplied with a factor $\mathrm{e}^{-d}$ with decay parameter $d$.

All intervals that are assigned to the methylated state feature a joint simulation parameter $P_{\text {meth }}$ for the probability of observing a methylated read by sequencing. Unmethylated regions have an analogous parameter $P_{\text {un }}$. Unmethylated regions have probability $P_{\text {un }}$ close to zero, methylated regions have $P_{\text {meth }}$ close to one. The probabilities for the intermediate states $S_{2}$ and $S_{4}$ are given by the arithmetic mean $0.5 P_{\text {un }}+0.5 P_{\text {meth }}$ in the WGBSSuite. Moreover, there are two so-called 'error' parameters $E_{\mathrm{un}}$ and $E_{\text {meth }}$ that control fluctuating probabilities for the measurements. Again, the error parameters for the intermediate states $S_{2}$ and $S_{4}$ are calculated by the arithmetic mean. Similarly, there are two parameters for the mean number of reads in methylated and unmethylated regions termed $M_{\text {meth }}$ and $M_{\text {un }}$. The mean number of reads for the intermediate states is again given by the average of both parameters.

For simulating differential methylation, a fraction $F$ of the intervals with a specific methylation state are drawn and then assumed as differentially methylated. Then, the direction of differential methylation is drawn according to a so-called 'balance' parameter (Rackham et al., 2015). The balance parameter was set to 0.5 which corresponds to equal probabilities for up- and downregulation. All simulation parameters are summarized in Supplementary Tables S1 and S2. The magnitudes of the differential methylation levels, i.e. the difference of the probabilities $P_{\text {meth }}$ or $P_{\text {un }}$ are used as effect size that is evaluated over the whole range for assessing the power of the approaches. 


\subsection{Optimizing simulation parameters}

To simulate the data realistically, the simulation parameters of the WGBSSuite were calibrated to our experimental datasets. For quantifying similarity of simulated and experimental data, we characterized datasets by calculating several attributes. At this point, it is important to characterize methylated as well as nonmethylated regions independently. To be able to discriminate regions with low methylation levels from high levels, a custom smoothing approach was applied as described in the Supplementary information.

For regions with high methylation levels that are termed presumably methylated (PM) in the following, and for regions that are presumably unmethylated (PU), 16 attributes were calculated:

1. Mean and $\mathrm{SD}$ of the number of reads ( $\Rightarrow 2$ attributes for PM, 2 attributes for PU).

2. Mean and SD of the methylation levels ( $\Rightarrow 2$ attributes for PM, 2 attributes for PU).

3. Mean and SD of the $\log _{10}$ distances of read positions $(\Rightarrow 2$ attributes).

4. Mean and SD of the $\log _{10}$ lengths of PM regions according to our smoothing approach ( $\Rightarrow 2$ attributes).

5. Mean and SD of the $\log _{10}$ lengths of PU regions $(\Rightarrow 2$ attributes).

6. Smoothing threshold for the number of reads ( $\Rightarrow 1$ attribute).

7. Smoothing threshold for the methylation level ( $\Rightarrow 1$ attribute)

Comparing the attributes $a_{1}^{\text {sim }}, \ldots, a_{16}^{\text {sim }}$ for the simulated data with the attributes $a_{1}^{\text {obs }}, \ldots, a_{16}^{\text {obs }}$ observed for real BS-seq data provided 16 residuals

$$
\operatorname{res}_{i}=\frac{a_{i}^{\mathrm{sim}}-a_{i}^{\mathrm{obs}}}{w_{i}}, i=1, \ldots, 16
$$

which were used to estimate 13 simulation parameters that have to be defined in the WGBSSuite. The weights $w_{1}, \ldots, w_{16}$ are required to ensure, that each residual enters with similar magnitude to the objective function to prevent that one attribute dominates. For calculating these weights, we uniformly drew 100 parameter vectors $\rightarrow \theta$ and calculated 100 residual vectors and calculated deviations as indicators for the typical spread of each attribute. The weights $w_{i}$ were then defined as the inverse of these SDs to obtain residuals res $i$ with a typical size of about 1 .

To prevent ill-conditioning, the residuals in (1) were augmented with additional regularizing residuals

$$
\operatorname{res}_{i}^{\text {prior }}=\frac{\theta_{i}-\theta_{i}^{\text {prior }}}{\operatorname{SD}\left(\theta_{i}^{\text {prior }}\right)}, i=1, \ldots, 13
$$

which can be interpreted as weak Gaussian priors for each of the 13 simulation parameters resulting in a total of 29 residuals. The target values of these penalties for $\theta_{i}$ are given by the average $\theta_{i}^{\text {prior }}$ $=0.5 U_{i}+0.5 L_{i}$ between upper $U_{i}$ and lower bounds $L_{i}$ and the priors' $\operatorname{SDs} \operatorname{SD}\left(\theta_{i}^{\text {prior }}\right)=U_{i}-L_{i}$ was chosen equal to the parameter ranges.

\subsubsection{Least-squares optimization}

$$
\hat{\theta}=\arg \min _{\theta} V(\theta) \text { with } V(\rightarrow \theta)=\sum_{i=1}^{29} \operatorname{res}_{i}^{2}
$$

using Matlab's trust region nonlinear least-squares optimization algorithm lsqnonlin (Coleman and Li, 1996) was then performed as described in the Supplementary. Supplementary Table S1 summarizes the simulation parameters as well as the assumed upper and lower bounds. The WGBSSuite was originally implemented in $\mathrm{R}$ (Rackham et al., 2015). Since evaluating the objective function in Matlab and calculating the residuals in $\mathrm{R}$ was too time-consuming for optimization with respect to run-time, we re-implemented the core of the WGBSSuite in Matlab by translating the code line by line. Optimization of the simulation parameters was performed in Matlab because optimization of the nonsmooth, stochastic objective function (3) is a numerically challenging task and it is known that optimization algorithms implemented in Matlab have strong performance (Raue et al., 2013). The original implementation in the WGBSSuite simulates methylation differences with constant magnitude. We added the functionality of changing the level of methylation differences continuously. The change of methylation probabilities was randomly drawn according to a uniform distribution $\pm U\left(0, \Delta_{\max }\right)$ in the range between 0 and $\Delta_{\max }=0.5$.

\subsection{Assessing DMR predictions}

The performance of DMR predictions was assessed in terms of precision and recall. For this evaluation, the number of correctly/incorrectly classified DNA positions was counted using the simulation data generated after calibrating the simulation parameters to individual datasets. For such simulated data, the underlying true differential methylation is known which enables assessment in terms of precision and recall. Recall is a synonym for sensitivity and is equivalent to the true-positive rate

$$
\text { recall }=T P / P
$$

that is given by the ratio of the number $T P$ of sites which were correctly classified as part of DMRs, relative to the total number of positives $P=T P+F N$, i.e. relative to the number of sites in all predicted DMRs.

Precision is a synonym for the positive predictive value and is defined as the fraction of predictions that are correct

$$
\text { precision }=\frac{T P}{T P+F P},
$$

i.e. the true positives relative to the sum of true positives and false positives $F P$. In our setup, the false positives $F P$ coincide with the number of sites which are in predicted DMRs but are in fact not within true DMRs. The F1-score is defined as the harmonic mean

$$
\frac{1}{\mathrm{~F} 1}=\frac{1}{2}\left(\frac{1}{\text { precision }}+\frac{1}{\text { recall }}\right)
$$

of precision and recall which is equivalent to

$$
\mathrm{F} 1=\frac{2 T P}{2 T P+F P+F N}
$$

because $P=T P+F N$ where $F N$ denotes false negatives, i.e. differentially methylated genomic positions that are not within predicted DMRs.

Because strong differences are easier to detect, the performance in general depends on the underlying magnitude of the differential methylation. Since this magnitude is unknown, we applied a powercalculation approach with two groups of samples, each with three replicates and evaluated the dependency of the F1-score on the true underlying magnitude of differential methylation. For this purpose, the simulated DMRs were sorted according to the true differential methylation level $\Delta$ and $\mathrm{F} 1(\Delta)$ was calculated for all DMRs which have at least a differential methylation level $\Delta$.

In this analysis a favorable approach is indicated by a superior curve

$$
\mathrm{F} 1_{\text {algorithm } \mathrm{A}}(\Delta)>\mathrm{F} 1_{\text {algorithm } \mathrm{B}}(\Delta) \Rightarrow \mathrm{A} \text { better than } \mathrm{B}
$$

because such an algorithm enables a more reliable identification of DMRs with differential levels up to $\Delta$.

\section{Results}

\subsection{Study design}

The major goal of this study is to compare selected DMR callers in a realistic performance comparison using simulated datasets based on real data. Therefore, a blinded study design was chosen, i.e. the 
DMR tools were applied to simulated BS-seq data without knowledge about the data. Blinded study designs are very common in other fields of research, e.g. for clinical studies. Recently, they were demanded for benchmark studies in the field of computation biology (Peters et al., 2018; Weber et al., 2019). The advantages of blinded benchmark studies are discussed in Boulesteix et al. (2017) and Kreutz (2016). Experimental data taken from different plant species served as templates for simulating realistic data. Characterization, calibration of the simulation parameters and generation of realistic simulated datasets was performed. For each experimental dataset (as summarized in Table 1), a corresponding simulation dataset with $1 \mathrm{e} 6$ genomic positions and three replicates in two groups was generated by Group I (in Freiburg). Subsequently, the simulated datasets were analyzed with selected DMR callers in a blind way by Group II (in Marburg). No information about the frequency or magnitudes of methylation or differential methylation was provided. In such a setting, each parameter of the individual algorithms has to be chosen as the suggested default or is adapted based on plausibility arguments or by manual inspection of preliminary outcomes like in real application settings. This strategy enables a realistic and almost unbiased scoring, because tuning of configuration parameters for improving the outcomes is prohibited by the study design. After analysis of the simulated data, the predicted DMRs for the evaluated tools were sent back to Group I to be compared with the true DMRs. Then, the outcomes were assessed by calculating F1-scores and by ranking the methods. The experimental data templates as well as the simulated datasets are publicly available at https://github.com/kreutz-lab/ DMR-DecisionTree.

\subsection{Inclusion and exclusion of DMR approaches}

After generating first simulated datasets, the blinded analyses were performed in Marburg between August 2017 and September 2018. For our study, we selected the DMR approaches that were available at that time and had been applied according to our knowledge in the context of plants. A special characteristic of plants is that DNA methylation occurs at $\mathrm{CHH}$ motifs. Since this motif is not symmetric, methylation is asymmetric on both DNA strands in contrast to methylation at $C G$ and $C H G$ residues. We, therefore, excluded BiSeq (Hebestreit et al., 2013), BEAT (Akman et al., 2014), DSS (Feng et al., 2014), RnBeads (Assenov et al., 2014), M3D (Mayo et al., 2015) because it has been claimed that they cannot handle methylation in non-CG context (Catoni et al., 2018).

After defiant was published (Condon et al., 2018) in February 2018, we decided to include defiant because of the performance benefits observed in Condon et al. (2018) and since the approach relies on reasonable statistical foundations. After information about the underlying truth and about the performances of the approaches had been shared, we could not include additional approaches since this would have violated our blinded study design.

\subsection{F1-scores}

We first analyzed the dependency of the chosen F1-scores on the true underlying methylation level as described in Section 2.5. Figure 1A shows that on average metilene and DMRcate are superior over the whole range. The horizontal axis corresponds to the parameter $\Delta$ which controls the probability difference of observing a methylated read in the WGBSSuite. $\Delta=0.5$, as an example, corresponds to a probability difference equal to 0.5 between the two groups of samples. The individual power curves for each algorithm and each dataset are shown as Supplementary Figure S6. Figure 1B shows a boxplot of the F1-scores for the 21 data contexts after averaging over the whole $\Delta$ range. The boxes indicate $25 \%$ and $75 \%$ percentiles, the lines denote the whole range of the F1-scores except outliers. This indicates that even for the best-performing methods, there are few datasets with inferior performance.

\subsection{Multivariate analysis}

The performance in terms of the F1-score for a simulated dataset depends on two major effects, the chosen DMR algorithm as well as on the simulation data context. The simulation dataset reflects attributes of the datasets which are in our setting determined from the experimental datasets used as templates for realistic simulation. To analyze and disentangle both effects, a linear model

$$
\mathrm{F} 1_{a d}=I+A_{a}+D_{d}+\varepsilon_{a d}, a=2, \ldots, 8 ; d=2, \ldots, 21
$$

for the F1-score observed for algorithm $a$ and data context $d$ was used to estimate the impact $A_{a}$ of the individual algorithms as well as the impact $D_{d}$ of the data attributes. As intercept $I$, the bestperforming approach (metilene) and the Phypa-R-CG data context was chosen. The outcome of the multivariate analysis is provided as Supplementary Table S3. The estimate $\hat{I}=0.75$ represents a reference F1-score. In this parameterization, the other estimated effects denote changes added to this intercept. $\varepsilon_{a d}$ represents unexplained Gaussian noise. The regression model (9) can be used to assess the significance of performance differences between DMR approaches while accounting/adjusting for performance differences originating from data characteristics.

Figure 2 shows the estimated effects and $95 \%$ confidence intervals. The intercept $\hat{I}=0.75$ is highlighted by gray shading. Negative effects indicate loss of performance on average compared to the reference analysis represented by intercept. The performance loss for Defiant $(-0.12$ with $P=0.026)$ and BSmooth $(-0.16$ with $P=0.004)$ is significant. For methylKit $(-0.27$ and $P=3.9 \mathrm{e}-6)$, MethylSig $(-0.35$ with $P \leq 1 \mathrm{e}-8)$ and MOABS (-0.22 with
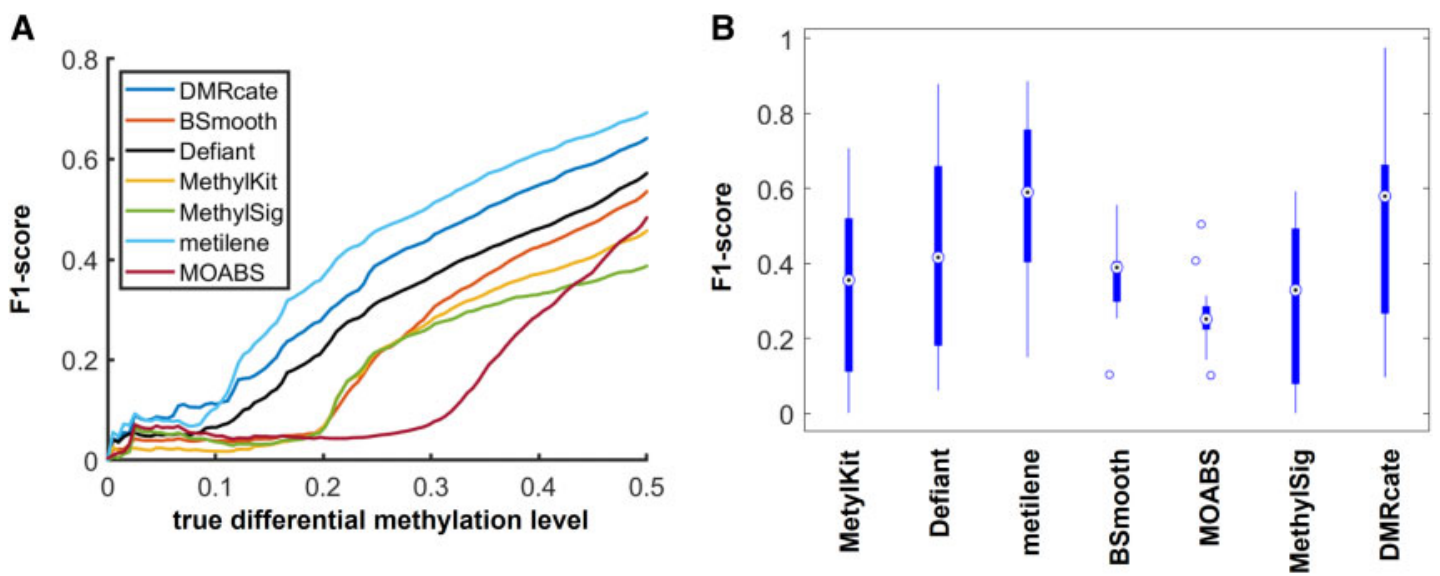

Fig. 1. (A) The increase in the F1-scores with the true differential methylation level. For this depiction, the outcomes of all data contexts were analyzed jointly. Metilene (light blue) and DMR cate (blue) exhibit superior performances over the whole range. (B) The distribution of the F1-scores averaged over all true differential levels $\Delta$ for the 21 individual datasets as boxplot. Again, DMRcate and metilene outperform the other approaches 
$P=1 \mathrm{e}-4)$ the estimated performance losses in terms of the F1score are in the range -0.22 to -0.35 and are very significant. The estimates for the different data contexts indicate the impact on the F1-score originating from the experimental data template. Note that in general increasing the sample size enhances significance of real performance differences. Therefore, an increased number of simulated and analyzed benchmark datasets could lead to further significant effects that might be too small to be significant for our chosen sample size. Despite remarkable effects, there seems to be no general tendency with respect to DNA context. By trend, datasets from Ae.arabicum yielded a performance decline compared to other plants, probably based on the much lower coverage of these datasets (Table 1). The comprehensive results of this statistical analysis are available as Supplementary Table S4.

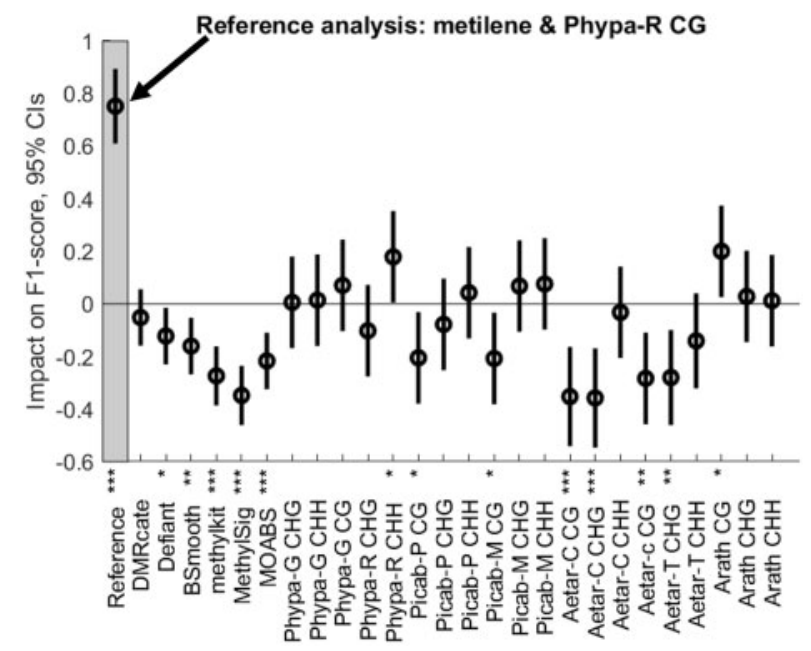

Fig. 2. Estimated impact on the F1-score of the individual algorithms and of the data contexts. Metilene as superior method has been chosen as a reference point (gray shading). For Phypa-R-CG context, an F1-score equals to $\hat{I}=0.75$ is obtained. The other black dots indicate the change of the F1-score if the algorithm is and/or the background is switched. This means that negative values (below the black horizontal line) decreases the performance. Error bars indicate $95 \%$ confidence intervals, i.e. all bars that do not cross the black line are significant according to a $95 \%$ significance level. Effects that are significantly different from zero are indicated by $* P<0.05, * P<0.01$ and $* * P<0.001$. Hence, all algorithms except $D M R$ cate are significantly worse on average over all data contexts

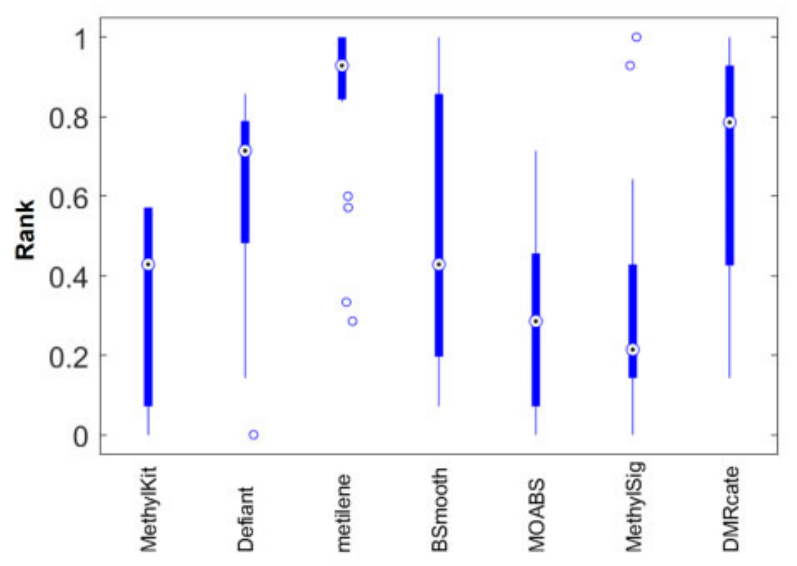

Metilene that had on average the best performance is also faster than most other tools on average. Only Defiant is clearly faster and methylKit exhibits a minor benefit in terms of computation times on a linux computer with Intel(R) Xeon(R) CPU E5-2609-0 2.40 GHz and 62.9 GB RAM. Details about runtimes and memory usage are provided as Supplementary information.

\subsection{Ranking of the algorithms and selection guidelines}

As a next step for deriving general rules for selecting a DMR approach, we calculated ranks over the methods' F1-scores for each data context. Figure 3 shows ranks of the algorithms as boxplot in panel (A) and as heatmap in panel (B). In our notation, a rank equals to one corresponds to the best performance, the least-performing approach has rank zero. metilene shows superior performance, but there are four outliers with inferior performance. The second bestperforming approach is DMRcate.

The black rectangles in Figure $3 \mathrm{~B}$ indicate four data contexts where metilene is not among the two best-performing approaches, all of them from Ae.arabicum. BSmooth performs well for these four datasets and is, therefore, a well-suited alternative for these data contexts, although it has inferior performance on average. Therefore, one strategy for properly choosing high-performing DMR methods would be using metilene by default and switching to $B S m o o t h$ for such special cases.

There is one setting, Aetar-C-CHH (first row in Fig. 3B) where this strategy has only medium performance (ranks for metilene and BSmooth are 0.28 and 0.57). DMRcate performs optimal for this dataset and can serve also as substitute for a second data (Aetar$\mathrm{T}-\mathrm{CHH}$ ) set where metilene performs bad ( $\mathrm{rank}=0.33$ ). Therefore, our suggested strategy is using metilene as default and switching to BSmooth or DMRcate. Choosing among these three algorithms always yields optimal performance with a superior rank (see Supplementary Fig. S5).

For generalizing this outcome, we identified data attributes as predictors for the performance and for guiding algorithm selection. We investigated the same data attributes that were used to characterize similarity between simulated and experimental data to derive a decision tree for optimal algorithm selection. Figure 4 shows the resulting data attributes which indicate optimal selection between two algorithms (Fig. 4A; metilene and BSmooth) or between three algorithms (Fig. 4B; metilene, BSmooth and DMRcate) which is our recommendation. At https://github.com/kreutz-lab/DMR-DecisionTree, we provide a Matlab implementation of the calculation of the data attributes and for evaluating this decision tree. This implementation can be

Fig. 3. (A) The ranking of the methods (best $=1$, worst $=0$ ) over each data context as boxplots. For this depiction, ranks were calculated via $R_{\text {method }}=$ $\operatorname{mean}_{i}\left(\operatorname{rank}_{\text {methods }}\left(F 1\left(\Delta_{i}\right)\right)\right)$ i.e. ranks were calculated for all methods along the evaluated methylation differences $\Delta_{i}$. Metilene shows superior performance with a median rank equals to 0.93 . The depiction as heatmap shown in B indicates that there are data context (highlighted by the black boxes) where metilene has inferior performance. This observation demands for an algorithm selection guideline based on attributes of the analyzed dataset. There are seven white colors indicating datasets where MethylSig or methylKit could not complete because of too low coverage. (Color version of this figure is available at Bioinformatics online.) 
A

Selection between two algorithms

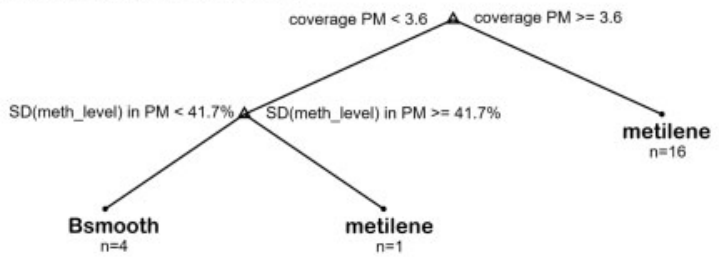

B

Selection between three algorithms

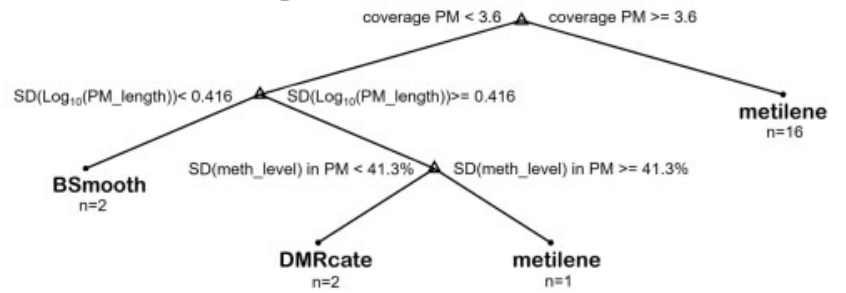

Fig. 4. Two decision trees based on data attributes indicating optimal selection between two and three approaches. (A) The selection guideline derived to choose between two algorithms. Metilene as the overall best algorithm is the default. For settings where metilene has bad performance, BSmooth serves as replacement. The decision tree indicates that in the case where the average coverage in PM regions is smaller than 3.6 and the measured methylation levels have large variability, BSmooth should be selected according to our analyses. (B) The optimal selection guideline which requires a data-based selection between three methods, namely between metilene by BSmooth and DMRcate. Again, metilene can be considered as default. BSmooth is selected for low coverage in PM $(<3.6)$ and small SD of the length of PMs $(<0.416)$. DMRcate is selected for low coverage in PM $(<3.6)$, large SD of the length of PMs $(>0.416)$ and small SD of the measured methylation levels $(<41.3 \%)$. If methylation levels within PMs are consistently increased, this SD is small. If methylation levels within PMs are consistently increased over all read positions in the region, this SD is small. Conversely, it is large if only a subset of the read positions in the region indicate methylation

applied to any new BS-seq dataset to predict the best-performing DMR approach based on our study.

\section{Discussion and summary}

After decades of rapid progress in the development of highthroughput experimental techniques, and establishment of computational methods for analyzing high-throughput data in parallel, a large number of computational algorithms and software packages for statistical analysis of omics-data are nowadays available. Most of the few available benchmark studies failed to derive generally superior algorithms because context-dependency is a common feature for benchmarking of computational methods. Nevertheless, context-specific application limits and the relationships between data characteristics and performance advantages are widely unknown. Therefore, it is typically difficult or even impossible to select optimally performing computational methods in applications for analysis of high-throughput data. Usage of suboptimal or inappropriate approaches, in turn, can lead to insignificant, misleading or even erroneous results and conclusions. Therefore, guidelines for the selection of computational methods that reliably and efficiently work in a given context are an important requirement for reliable research and for the transfer of computational methods to experimental practice.

In this study, we present a benchmark study comprising seven prominent methods for identification of DMRs were applied for the plant methylation contexts: $C G, C H G$ and $C H H$. We tested DMR algorithms for 21 experimental datasets from plants belonging to three taxonomic groups. Calibration of the simulation parameters individually to each experimental dataset enabled simulation of realistic benchmark data. Previous benchmark studies provide only heterogeneous and fragmentary information for plants. Catoni et al. (2018) assessed the performance of MethCP, their own new approach, with MethlySig and methylKit for an A.thaliana experiment where the differential methylation between wild-type and methyltransferase knockout plants was calculated. Methyltransferase knockouts block methylation almost entirely and, therefore, cause uniform and unphysiological levels of underlying methylation differences. Moreover, they observed inconsistent outcomes for CG, $\mathrm{CHG}$ and $\mathrm{CHH}$, that diminished the utility of that study for our purposes. In fact, most studies in the literature comparing the performance of DMR approaches were performed by jointly presenting a new approach. Such studies, however, are easily biased because they are not independent (Boulesteix et al., 2013, 2018) and they are at least partly performed to demonstrate benefits of the new method they are presenting.

In contrast to previous studies, we tried to design a benchmark study which has minimal bias and is tailored to DMR data from plants. In our study, the DMR approaches were applied to simulated data but without any knowledge about the true differential methylation like in real application settings. This blinded study design provides an assessment in terms of precision and recall which is comparable to application settings where configurations parameters have to be selected based on default suggestions and manual inspection of preliminary outcomes. One shortcoming of the blinded study design is that it is not possible to extend the study by additional methods because this prohibits blinded configurations and evaluations of the methods. Therefore, we could not add other DMR approaches after the first assessments were conducted.

Our benchmark study showed clear advantages of some methods although no approach outperformed all other methods for all datasets. Overall, metilene exhibited superior performance and we suggest usage of metilene as default approach. However, we also found few data settings where metilene has inferior performance. This is in agreement with previous studies (Condon et al., 2018; Gong and Purdom, 2018; Jühling et al., 2016). We found that for our datasets, where metilene showed lower performance, either DMRcate or $B S m o o t h$ were superior and could, therefore, serve as better options. In agreement with Condon et al. (2018), Defiant also performed well in our setting but the performance of metilene was superior.

We could find data attributes like coverage or lengths of PM regions that guide to the optimal algorithm choice for all our datasets. We provide publicly available code that can be used to calculate these attributes. We derived a decision tree based on these attributes indicating the optimal choice. The optimal rule derived for our scope use metilene as default and guides the usage of BSmooth and DMR cate as substitutes. BSmooth should be selected in case of small coverage and small variation of the lengths of DMRs. DMRcate is the method of choice for small coverage, large variation of the length of DMRs and small SD of the measured methylation levels.

Analogous guidelines are common in some traditional statistical fields, e.g. for selecting statistical tests. As an example, for twogroup comparison, the $t$-test has superior power for normally distributed errors, but it is suggested to use the Wilcoxon rank-sum test as substitute in case of unknown noise distribution or if the data contains outliers. Such rules are an important requirement for reliability of research in all bioinformatics fields for the transfer of new computational approaches to basic research as well as clinical and industrial applications.

Our outcomes are in line with the common tendency in the literature that for comprehensive computational analysis of highthroughput data there is usually not a single outperforming approach (Shippy et al., 2006; Su et al., 2014; Webb-Robertson et al., 2015). Instead, the performances depend on attributes of the analyzed data. These insights demand for algorithm selection guidelines based on attributes of the data.

A relevant remaining question is a common issue for all benchmark studies, namely to which extent the outcomes translate into other applications and biological contexts. Since BS-seq is a generic experimental technique that is applicable independently of the biological background, the question reduces to generalization and 
validity for new datasets with distinct attributes. The only solution to this universal issue is emphasizing the importance of comprehensive benchmark studies, establishment of standards for the designs of benchmark studies as well as a broad set of representative benchmark problems that are routinely utilized for development of new algorithms. The benchmark data which was generated within this study contributes to these efforts.

In terms of the data attributes that we used for characterizing our experimental data and for optimizing the similarity of the simulated datasets, we observed that the differences between the three DNA contexts ( $C G, C H G$ and $\mathrm{CHH}$ ) are larger than the differences between the different plants (see Supplemental Information, Fig. 4). This observation in combination with the fact that we investigated plants from three different taxonomic groups indicates that the data used in our study samples a broad range of BS-seq data characteristics, thus we expect rather general outcomes. This speculation, however, can only be proven if larger sets of different organisms are evaluated which is beyond the scope of this study.

Our study pinpoints four important general aspects for performing meaningful benchmark studies. (i) We chose a blinded design to diminish biased assessment. (ii) The study is independent since none of the authors were involved in the development of any DMR methods tested. (iii) A multivariate statistical model has been applied for assessing significance and for decomposing the impact of algorithm selection from other effects. Moreover, (iv) a data-based decision rule has been derived to select between competing computational methods which is applicable to any new dataset.

\section{Acknowledgements}

We also acknowledge funding by the European Research Area Network for Coordinating Action in Plant Sciences (ERA-CAPS) for the 'SeedAdapt' consortium project (https://www.seedadapt.eu), and the Austrian Science Fund FWFI3979.

\section{Funding}

This work was supported by the German Ministry of Education and Research [EA:Sys FKZ031L0080] and by the German Research Foundation (DFG) under Germany's Excellence Strategy [CIBSS-EXC-2189-2100249960390939984]. Parts were funded by the European Training Network EpiDiverse (https://epidiverse.eu) that received funding from the EU Horizon 2020 program under Marie Skłodowska-Curie [agreement number 764965]. We also acknowledge funding by the European Research Area Network for Coordinating Action in Plant Sciences (ERA-CAPS) for the 'SeedAdapt' consortium project (https://www.seedadapt.eu), and the Austrian Science Fund FWFI3979.

Conflict of Interest: none declared.

\section{References}

Akalin,A. et al. (2012) methylKit: a comprehensive R package for the analysis of genome-wide DNA methylation profiles. Genome Biol., 13, R87.

Akman,K. et al. (2014) Genome-wide quantitative analysis of DNA methylation from bisulfite sequencing data. Bioinformatics, 30, 1933-1934.

Assenov,Y. et al. (2014) Comprehensive analysis of DNA methylation data with RnBeads. Nat. Methods, 11, 1138-1140.

Bewick,A.J. et al. (2017) Chromomethylases and gene body DNA methylation in plants. Genome Biol., 18, 65.

Bewick,A.J. and Schmitz,R.J. (2017) Gene body DNA methylation in plants. Curr. Opin. Plant Biol., 36, 103-110.

Boulesteix,A.L. et al. (2013) A plea for neutral comparison studies in computational sciences. PLoS One, 8, e61562.

Boulesteix,A.L. et al. (2017) Towards evidence-based computational statistics: lessons from clinical research on the role and design of real-data benchmark studies. BMC Med. Res. Methodol., 17, 138.

Boulesteix,A.-L. et al. (2018) On the necessity and design of studies comparing statistical methods. Biom. J., 60, 216-218.

Catoni,M. et al. (2018) DMRcaller: a versatile R/Bioconductor package for detection and visualization of differentially methylated regions in $\mathrm{CpG}$ and non-CpG contexts. Nucleic Acids Res., 46, e114.
Chen,X. et al. (2018) ChIP-seq: a powerful tool for studying protein-DNA interactions in plants. Mol. Biol., 27, 171-180.

Coleman,T. and Li,Y. (1996) An interior, trust region approach for nonlinear minimization subject to bounds. SIAM J. Optimiz., 6, 418-445.

Condon,D.E. et al. (2018) Defiant: (DMRs: easy, fast, identification and ANnoTation) identifies differentially methylated regions from iron-deficient rat hippocampus. BMC Bioinformatics, 19, 31.

Dolzhenko,E. and Smith,A.D. (2014) Using beta-binomial regression for high-precision differential methylation analysis in multifactor whole-genome bisulfite sequencing experiments. BMC Bioinformatics, 15, 215.

Feng,H. et al. (2014) A Bayesian hierarchical model to detect differentially methylated loci from single nucleotide resolution sequencing data. Nucleic Acids Res., 42, 669.

Gong,B. and Purdom,E. (2018) MethCP: differentially methylated region detection with change point models. bioRxiv, doi:http://dx.doi.org/10.1101/ 265116.

Hansen,K.D. et al. (2012) BSmooth: from whole genome bisulfite sequencing reads to differentially methylated regions. Genome Biol., 13, R83.

Hebestreit,K. et al. (2013) Detection of significantly differentially methylated regions in targeted bisulfite sequencing data. Bioinformatics, 29, $1647-1653$.

Heer,K. et al. (2018) Detection of somatic epigenetic variation in Norway spruce via targeted bisulfite sequencing. Ecol. Evol., 8, 9672-9682.

Jühling,F. et al. (2016) Metilene: fast and sensitive calling of differentially methylated regions from bisulfite sequencing data. Genome Res., 26, 256-262.

Kreutz,C. (2016) New concepts for evaluating the performance of computational methods. IFAC-PapersOnLine, 49, 63-70.

Lang,D. et al. (2008) Exploring plant biodiversity: the Physcomitrella genome and beyond. Trends Plant Sci., 13, 542-549.

Lang,D. et al. (2018) The Physcomitrella patens chromosome-scale assembly reveals moss genome structure and evolution. Plant J., 93, 515-533.

Liu,Y. et al. (2019) Bisulfite-free direct detection of 5-methylcytosine and 5-hydroxymethylcytosine at base resolution. Nat. Biotechnol., 37, 424-429.

Mayo,T.R. et al. (2015) M3D: a kernel-based test for spatially correlated changes in methylation profiles. Bioinformatics, 31, 809-816.

Meyberg,R. et al. (2019) Characterization of evolutionarily conserved key players affecting eukaryotic flagellar motility and fertility using a moss model. bioRxiv, doi:https://doi.org/10.1101/728691.

Park,Y. et al. (2014) MethylSig: a whole genome DNA methylation analysis pipeline. Bioinformatics, 30, 2414-2422.

Peters,B. et al. (2018) Putting benchmarks in their rightful place: the heart of computational biology. PLoS Comput. Biol., 14, e1006494.

Peters,T.J. et al. (2015) De novo identification of differentially methylated regions in the human genome. Epigenet. Chromatin , 8, 6 .

Rackham,O.J.L. et al. (2015) WGBSSuite: simulating whole-genome bisulphite sequencing data and benchmarking differential DNA methylation analysis tools. Bioinformatics, 31, 2371-2373.

Raue,A. et al. (2013) Lessons learned from quantitative dynamical modeling in systems biology. PLoS One, 8, e74335.

Robinson,M.D. et al. (2014) Statistical methods for detecting differentially methylated loci and regions. Front. Genet., 5, 324.

Sahu,P.P. et al. (2013) Epigenetic mechanisms of plant stress responses and adaptation. Plant Cell Rep., 32, 1151-1159.

Seymour,D.K. et al. (2014) Evolution of DNA methylation patterns in the Brassicaceae is driven by differences in genome organization. PLoS Genet., 10, e1004785.

Shippy, R. et al. (2006) Using RNA sample titrations to assess microarray platform performance and normalization techniques. Nat. Biotechnol., 24, 1123-1131.

Su,Z. et al. (2014) A comprehensive assessment of RNA-seq accuracy, reproducibility and information content by the sequencing quality control consortium. Nat. Biotechnol., 32, 903.

Sun,D. et al. (2014) MOABS: model based analysis of bisulfite sequencing data. Genome Biol., 15, R38.

Webb-Robertson,B.-J.M. et al. (2015) Review, evaluation, and discussion of the challenges of missing value imputation for mass spectrometry-based label-free global proteomics. J. Proteome Res., 14, 1993-2001.

Yang,Y. and Scott,S.A. (2017) DNA methylation profiling using long-read single molecule real-time bisulfite sequencing (SMRT-BS). Methods Mol. Biol., 1654, 125-134.

Weber,L.M. et al. (2019) Essential guidelines for computational method benchmarking. Genome Biol., 20, 125. 